For Rapid Communications

\title{
Function of Sox2 in ependymal cells of lesioned spinal cords in adult zebrafish
}

Kazuhiro Ogai $^{\mathrm{a}, \mathrm{b}}$, Kumi Nakatani ${ }^{\mathrm{c}}$, Suguru Hisano ${ }^{\mathrm{c}}$, Kayo Sugitani ${ }^{\mathrm{c}}$, Yoshiki Koriyama ${ }^{\mathrm{a}, \mathrm{d}}$, Satoru Kato ${ }^{\mathrm{a},{ }^{*}}$

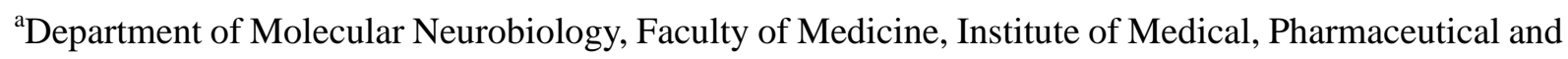
Health Sciences, Kanazawa University, 13-1 Takaramachi, Kanazawa, Ishikawa 920-8640, Japan

${ }^{\mathrm{b}}$ Wellness Promotion Science Center, Institute of Medical, Pharmaceutical and Health Sciences,

Kanazawa University, 5-11-80 Kodatsuno, Kanazawa, Ishikawa 9200942, Japan.

${ }^{\mathrm{c}}$ Department of Clinical Laboratory Science, Graduate School of Medical Science, Kanazawa

University, 5-11-80 Kodatsuno, Kanazawa, Ishikawa 920-0942, Japan

${ }^{\mathrm{d}}$ Faculty of Pharmaceutical Sciences, Suzuka University of Medical Science, 3500-3 Minamitamagaki, Suzuka, Mie 513-8670, Japan.

*All correspondence should be addressed to:

Satoru Kato

Department of Molecular Neurobiology, Graduate School of Medical Science, Kanazawa University,

13-1 Takaramachi, Kanazawa, Ishikawa 9208640, Japan

Tel.: +81 76265 2450; Fax: +81 762344235

E-mail address: satoru@med.kanazawa-u.ac.jp 


\section{Abstract}

The sex-determining region Y-box 2 (Sox2) is related not only to pluripotency, but also to cell proliferation. Zebrafish can regain their motor function after spinal cord injury (SCI). Following SCI, new motor neurons are produced from proliferating ependymal cells. Here, we investigated the expression and function of Sox2 after SCI in zebrafish. Sox2 was upregulated as early as 1 day post-lesion (dpl) in ependymal cells, which was followed by cell proliferation. Sox2 knockdown significantly decreased the number of proliferating cells at $5 \mathrm{dpl}$. The results of this study suggest a role of Sox2 as one of the proliferation initiators in ependymal cells after SCI.

Key words: zebrafish, spinal cord regeneration, sox2, cell proliferation 
Spinal cord injury (SCI) in mammals leads to the loss of motor neurons within the lesion site, without replacement or renewal (Pinto and Götz, 2007) and leads to neuronal cell death in the primary motor cortex (Hains et al., 2003). In contrast to such tragic events, adult zebrafish can regenerate motor neurons in the spinal cord and protect upper motor neurons in the brainstem against cell death via several regenerative mechanisms (Becker and Becker, 2007; Dias et al., 2012; Ogai et al., 2012). Given their high regenerative capacity, zebrafish have been considered to be a useful model to understand the molecular mechanisms that underlie spinal cord regeneration. For example, in the lesioned spinal cord of zebrafish, ependymo-radial glial cells, which correspond to the mammalian ependymal cells that line the ventricle, dedifferentiate and proliferate to produce new motor neurons; in turn, these cells finally exhibit terminal differentiation and integration into the pre-existing circuitry of spinal cord and muscular tissue (Reimer et al., 2008).

The X-linked sex-determining region Y-box 2 (Sox2) is famous for its ability to turn somatic cells into stem cells (Takahashi and Yamanaka, 2006); however, it is also related to neural development (Zappone et al., 2000; Ferri et al., 2004). Recently, Gaete et al. (2012) reported that Sox2 is upregulated in ependymal cells after spinal cord injury in larval, but not in adult, Xenopus. Moreover, Sox2 is reportedly upregulated in severed spinal cords of adult zebrafish that show greater regenerative capacity (Hui et al., 2014). Therefore, it is suggested that the level of Sox2 expression in ependymal cells is closely correlated with the regeneration capacity of lesioned spinal cords. The identification of Sox2 function during spinal cord regeneration in adult zebrafish, which has not yet been reported, will provide more specific insights into the effect of Sox2 on regenerative capacity in adult vertebrates. Therefore, in this study, we evaluated the expression and function of Sox2 in lesioned spinal cords of adult zebrafish. We showed the upregulation of Sox2 in ependymal cells located close to the lesion site soon after SCI. In addition, we demonstrated that the upregulation of Sox2 took place before the proliferation of ependymal cells and that the Sox2 knockdown in ependymal cells decreased the number of proliferating cells. These data suggest a role of Sox2 as a proliferation initiator. 
All experiments described below were approved by the Committee on Animal Experimentation of Kanazawa University, and all attention was paid to minimize pain and the number of fish used. Adult zebrafish (Danio rerio; 3-4 cm in body length) of either sex were used throughout this study. For SCI, fish were anesthetized by immersion in $0.033 \%$ ethyl 3-aminobenzoate methanesulfonic acid (MS-222;Sigma-Aldrich, MO, USA) in PBS for 5 min. A small incision was made at the side of the fish, and the spinal cord was completely transected $4 \mathrm{~mm}$ caudal to the brainstem-spinal cord junction using microscissors under visual control, followed by sealing of the wound using Histoacryl (B. Braun Melsungen AG, Melsungen, Germany)(Becker et al., 2004; Reimer et al., 2008). The fish were then reared in water at $28^{\circ} \mathrm{C}$ until appropriate time points.

For mRNA extraction,the fish were euthanized by an overdose (0.1\%) of MS-222 for 10 min, and spinal cords within $5 \mathrm{~mm}$ around the lesion site were collected from at least 3 fish per time point. Total RNA was extracted using Sepasol-RNA I Super G (Nacalai Tesque, Kyoto, Japan), according to the manufacturer's instructions. Single-stranded cDNAs were then synthesized using the ReverTra Ace reverse transcription enzyme (Toyobo, Osaka, Japan). RT-PCR of the sox2 mRNA in zebrafish spinal cords was conducted using zebrafish sox2-specific primers (forward, 5'-CGC TCC AGT ACA ACT CCA TGA CC-3'; reverse, 5'-TTA CAT ATG CGA TAA GGG AAT CGT GC-3') and SapphireAmp Fast PCR Master Mix (Takara, Shiga, Japan). The resultant sox2 PCR products were electrophoresed on agarose gels, visualized with ethidium bromide, and captured using the GeneGenius 2 Bio Imaging System (Syngene, MD, USA). Semi-quantitative densitometrical analyses were conducted using the ImageJ software (National Institute of Health, MD, USA).

For immunohistochemistry and in situ hybridization, spinal cords of euthanized fish were fixed with $4 \%$ paraformaldehyde/PBS overnight at $4^{\circ} \mathrm{C}$. On the next day, $50-\mu \mathrm{m}$-thick transversal vibratome sections of spinal cords within $750 \mu \mathrm{m}$ around the lesion site were prepared. Immunohistochemistry against Sox2 (1:500; GeneTex, CA, USA) and/or PCNA (1:1000; 
Sigma-Aldrich) was carried out as described previously (Dias et al., 2012) using appropriate Alexa Fluor 488- or 594-conjugated secondary antibodies (1:200; Life Technologies, CA, USA). In situ hybridization was also performed using a method described previously (Dias et al., 2012) with a zebrafish sox2-specific antisense cRNA probe. Immunoreaction and in situ hybridization images were acquired using a confocal microscope (LSM510; Carl Zeiss, Oberkochen, Germany).

Twosox2 morpholinos (MOs) [MO(1), 5'-GCT CGG TTT CCA TCA TGT TAT ACA T-3', Vivo-Porter coupled; MO(2), 5'-GAA AGT CTA CCC CAC CAG CCG TAA A-3' (Kamachi et al., 2008), Vivo-Porter coupled] were designed to block the expression of sox2 gene. A standard MO (5'-CCT CTT ACC TCA GTT ACA ATT TAT A-3', Vivo-Porter coupled) was used as a control (Veldman et al., 2007). All MOs were purchased from GeneTools, OR, USA. The MOs were applied to the lesion site of the spinal cord as described previously (Guo et al., 2011; Lin et al., 2012).

The number of PCNA-positive (PCNA ${ }^{+}$) cells was counted as described previously (Dias et al., 2012) and was calculated for the entire $1.5 \mathrm{~mm}$ surrounding the lesion site.

Values are represented as means \pm SEM. Statistical analyses of sox2 expression level and the number of Sox $2^{+}$cells were performed using Student's $t$-test and the one-way analysis of variance (ANOVA) followed by Tukey's post hoc test, respectively, using the Prism software (version 6.0c; GraphPad Software, CA, USA)

RT-PCR ofsox2 in the lesioned spinal cords showed that the expression of the sox2 mRNA was upregulated within $1 \mathrm{dpl}$, peaked at $3 \mathrm{dpl}$, and was sustained at a relatively high level up to $20 \mathrm{dpl}$ (Fig. 1A). At $3 \mathrm{dpl}$, the time at which the expression of sox 2 reached a peak, we observed a statistically significant increase in expression compared with unlesioned spinal cords (Fig. 1A; $p<0.01 ; n=4$ ). Next, we aimed to determine the localization of the upregulated sox2 mRNA and Sox2 protein following injury. As shown in Fig. 1B and C, the upregulated sox2 mRNA and Sox2 protein were detected predominantly around the ventricle, where ependymal cells exist, on both rostral and caudal sections to 
the injury site.

Given that Sox2 can activate cell proliferation (Tompkins et al., 2011) and that SCI induces the dedifferentiation and proliferation of ependymal cells to produce new motor neurons (Reimer et al., 2008), we next investigated the relationship between Sox2 expression and cell proliferation. At $1 \mathrm{dpl}$, Sox $2^{+}$cells appeared around the ventricle (Fig. 2A); however, none of these Sox $2^{+}$cells expressed PCNA (Fig. 2D, G). At 3 dpl, some of the Sox2 ${ }^{+}$cells became $\mathrm{PCNA}^{+}$, implying that Sox2 ${ }^{+}$cells re-entered the cell cycle (Fig. 2B, E, H). At 5 dpl, additional Sox2 ${ }^{+}$cells expressed PCNA compared with those observed at 3 dpl (Fig. 2C, F, I). Hence, the expression of Sox2 may precede cell proliferation.

To elucidate the direct relationship between Sox2 expression and cell proliferation, we employed sox2-targeted morpholinos (MOs) to knockdown the sox2 expression in the ependymal cells. Sox2 MO could indeed decrease the expression level of Sox2 (Fig. 3B) compared with control MO treatment (Fig. 3A) in ependymal cells. As a result, sox2 MO significantly, but not completely, reduced the number of PCNA ${ }^{+}$cells around the central canal (Fig. 3C-G; $p<0.001$ by one-way ANOVA; $n=4-6$ ) at $5 \mathrm{dpl}$. These results imply the role of Sox2 as one of the cell proliferation-related molecules following SCI.

After SCI in zebrafish, ependymal cells re-enter the cell cycle (i.e., they dedifferentiate), proliferate, migrate, and differentiate into mature motor neurons, to compensate for the motor neurons that are lost after the spinal lesion (Reimer et al., 2008; Reimer et al., 2009). Therefore, ependymal cells are an important source of new motor neurons, and the dedifferentiation and proliferation of ependymal cells is necessary for the production of new motor neurons and the regeneration of the spinal cord.

To the best of our knowledge, Sox2 is one of the earliest factors that are expressed in ependymal cells following injury, as we observed Sox2 upregulation as early as $1 \mathrm{dpl}$ (Figs. 1A and 2A). It has been reported that the proliferation (i.e., PCNA positivity) of ependymal cells starts at $3 \mathrm{dpl}$ 
(Reimer et al., 2008; Goldshmit et al., 2012), which is consistent with our PCNA profiles (Fig. 2). Together with the finding of this study that Sox2 ${ }^{+}$cells, but not proliferating $\left(\mathrm{PCNA}^{+}\right)$cells, were observed at $1 \mathrm{dpl}$ (Fig. 2A, D), these results suggest that Sox2 is an important factor related to cell proliferation. In fact, sox2 knockdown significantly decreased the number of PCNA ${ }^{+}$ependymal cells (Fig. 3). However, the number of proliferating cells was not completely depleted by sox2 knockdown. This can be explained by the existence of other proteins of the Sox family. It is known that Sox4 can compensate for the function of Sox11 in a zebrafish optic nerve injury model (Veldman et al., 2007). In addition, Guo et al. (2011) reported the upregulation of Sox11b in the lesioned zebrafish spinal cord. These authors also showed that sox $11 b$ knockdown significantly, but likewise not completely, decreased the number of proliferating cells. Therefore, it is speculated that Sox2 and other Sox-family proteins may function concomitantly toward ependymal cell proliferation.

Very recently, Hui et al. (2014) reported the upregulation of Sox2 in the lesioned zebrafish spinal cord, which is consistent with our findings. Our study may add the functional aspect of Sox2 via which the proliferation of ependymal cells might be triggered. We observed a decrease in GFAP (which is a marker of mature ependymal cells) immunoreactivity around the central canal at 3 dpl (data not shown), implying the dedifferentiation of mature ependymal cells following injury. Our next goal will be to identify the role of Sox2 in the dedifferentiation of ependymal cells, which may take place before their proliferation.

\section{Acknowledgements}

We would like to thank Ms. Sachiko Higashi and Ms. Tomoko Kano for their secretarial and technical assistance. This work was supported in part by JSPS KAKENHI Grants [No. 25890007 (K.O.) and 25640008 (S.K.)]. This study was also funded in part by the MEXT/JST Tenure Track Promotion Program (K.O.). 


\section{References}

Becker, C.G., Lieberoth, B.C., Morellini, F., Feldner, J., Becker, T., Schachner, M., 2004. L1.1 is involved in spinal cord regeneration in adult zebrafish. J. Neurosci. 24, 7837-7842.

Becker, C.G., Becker, T., 2007. Zebrafish as a Model System for Successful Spinal Cord Regeneration, In: Becker, C.G., Becker, T. (Eds.), Model Organisms in Spinal Cord Regeneration. Wiley VCH, Germany, pp. 289-313.

Dias, T.B., Yang, Y.J., Ogai, K., Becker, T., Becker, C.G., 2012. Notch signaling controls generation of motor neurons in the lesioned spinal cord of adult zebrafish. J. Neurosci. 32, 3245-3252.

Ferri, A.L., Cavallaro, M., Braida, D., Di Cristofano, A., Canta, A., Vezzani, A., Ottolenghi, S., Pandolfi, P.P., Sala, M., DeBiasi, S., Nicolis, S.K., 2004. Sox2 deficiency causes neurodegeneration and impaired neurogenesis in the adult mouse brain. Development 131, 3805-3819.

Gaete, M., Muñoz, R., Sánchez, N., Tampe, R., Moreno, M., Contreras, E.G., Lee-Liu, D., Larraín, J., 2012. Spinal cord regeneration in Xenopus tadpoles proceeds through activation of Sox2-positive cells. Neural development 7, 13.

Goldshmit, Y., Sztal, T.E., Jusuf, P.R., Hall, T.E., Nguyen-Chi, M., Currie, P.D., 2012. Fgf-dependent glial cell bridges facilitate spinal cord regeneration in zebrafish. J. Neurosci. 32, 7477-7492.

Guo, Y., Ma, L., Cristofanilli, M., Hart, R.P., Hao, A., Schachner, M., 2011. Transcription factor Sox11b is involved in spinal cord regeneration in adult zebrafish. Neuroscience 172, 329-341.

Hains, B.C., Black, J.A., Waxman, S.G., 2003. Primary cortical motor neurons undergo apoptosis after axotomizing spinal cord injury. J. Comp. Neurol. 462, 328-341.

Hui, S.P., Sengupta, D., Lee, S.G., Sen, T., Kundu, S., Mathavan, S., Ghosh, S., 2014. Genome wide expression profiling during spinal cord regeneration identifies comprehensive cellular 
responses in zebrafish. PLoS One 9, e84212.

Kamachi, Y., Okuda, Y., Kondoh, H., 2008. Quantitative assessment of the knockdown efficiency of morpholino antisense oligonucleotides in zebrafish embryos using a luciferase assay. Genesis $46,1-7$

Lin, J.F., Pan, H.C., Ma, L.P., Shen, Y.Q., Schachner, M., 2012. The cell neural adhesion molecule contactin-2 (TAG-1) is beneficial for functional recovery after spinal cord injury in adult zebrafish. PLoS One 7, e52376.

Ogai, K., Hisano, S., Mawatari, K., Sugitani, K., Koriyama, Y., Nakashima, H., Kato, S., 2012. Upregulation of anti-apoptotic factors in upper motor neurons after spinal cord injury in adult zebrafish. Neurochem. Int. 61, 1202-1211.

Pinto, L., Götz, M., 2007. Radial glial cell heterogeneity--the source of diverse progeny in the CNS. Prog. Neurobiol. 83, 2-23.

Reimer, M.M., Sörensen, I., Kuscha, V., Frank, R.E., Liu, C., Becker, C.G., Becker, T., 2008. Motor neuron regeneration in adult zebrafish. J. Neurosci. 28, 8510-8516.

Reimer, M.M., Kuscha, V., Wyatt, C., Sörensen, I., Frank, R.E., Knüwer, M., Becker, T., Becker, C.G., 2009. Sonic hedgehog is a polarized signal for motor neuron regeneration in adult zebrafish. J. Neurosci. 29, 15073-15082.

Takahashi, K., Yamanaka, S., 2006. Induction of pluripotent stem cells from mouse embryonic and adult fibroblast cultures by defined factors. Cell 126, 663-676.

Tompkins, D.H., Besnard, V., Lange, A.W., Keiser, A.R., Wert, S.E., Bruno, M.D., Whitsett, J.A., 2011. Sox2 activates cell proliferation and differentiation in the respiratory epithelium. Am. J. Respir. Cell Mol. Biol. 45, 101-110.

Veldman, M.B., Bemben, M.A., Thompson, R.C., Goldman, D., 2007. Gene expression analysis of zebrafish retinal ganglion cells during optic nerve regeneration identifies KLF6a and KLF7a as important regulators of axon regeneration. Dev. Biol. 312, 596-612. 
Zappone, M.V., Galli, R., Catena, R., Meani, N., De Biasi, S., Mattei, E., Tiveron, C., Vescovi, A.L., Lovell-Badge, R., Ottolenghi, S., Nicolis, S.K., 2000. Sox2 regulatory sequences direct expression of a (beta)-geo transgene to telencephalic neural stem cells and precursors of the mouse embryo, revealing regionalization of gene expression in CNS stem cells. Development 127, 2367-2382.

\section{Figure Legends}

Figure 1 Upregulation of the sox2 mRNA and Sox2 protein in the ependymal zone of lesioned zebrafish spinal cords. (A) The sox 2 mRNA was upregulated as early as 1 day post-lesion (dpl), peaked at $3 \mathrm{dpl}$, and was sustained at a relatively high level up to $20 \mathrm{dpl}$. The upregulation of the sox 2 mRNA was significant at $3 \mathrm{dpl}\left({ }^{* *} p<0.01, n=4\right)$. (B, C) The sox 2 mRNA and Sox 2 protein were located around the central canal, both rostral and caudal to the lesion site, at $3 \mathrm{dpl}$. The open arrowheads in (B) indicate pigmented epithelium. The dotted lines in (C) denote the central canal. Scale bars in (B) and (C), $50 \mu \mathrm{m}$.

Figure 2 Sox2 expression and cell proliferation following spinal injury. At 1 dpl, Sox2 was detected in the ependymal zone (A); however, Sox $2^{+}$cells were not positive for PCNA (D, G). At 3 dpl, additional Sox $2^{+}$cells appeared $(\mathbf{B})$, some of which were $\operatorname{PCNA}^{+}(\mathbf{E}, \mathbf{H})$. At 5 dpl, most Sox ${ }^{+}$cells became PCNA $^{+}(\mathbf{C}, \mathbf{F}, \mathbf{I})$. Thus, Sox2 was expressed in ependymal cells before their proliferation. The dotted lines denote the central canal. Scale bar in (A), $50 \mu \mathrm{m}$.

Figure 3 Sox2 was partially required for ependymal cell proliferation. (A, B) Immunoreactivity of Sox2 in ependymal cells was weaker in sox2 morpholino (MO)-treated spinal cord (B) compared with control MO-treated one (A) at $5 \mathrm{dpl}$. (C, D) Representative images of PCNA immunostaining at $5 \mathrm{dpl}$. A sox2-targeted MO reduced the number of $\mathrm{PCNA}^{+}$cells (D) compared with a standard MO (C). Panel (E) and (F) are merged images of (A) and (C), and (B) and (D), respectively. (G) Quantification of PCNA ${ }^{+}$ 
cells around central canal. Both sox2 $\mathrm{MO}(1)$ and $\mathrm{MO}(2)$ significantly, but not completely, reduced the number of PCNA ${ }^{+}$cells.n = 5, 6, 5, and 4 for lesion only, control MO, sox2 $\mathrm{MO}(1)$, and sox2 $\mathrm{MO}(2)$, respectively. ${ }^{*} p<0.05$ and ${ }^{* * *} p<0.001$ vs. the lesion only; $\dagger \dagger p<0.01$ and $\dagger \dagger \dagger p<0.001$ vs. control MO. n.s.: not significant. The dotted lines denote the central canal. Scale bar in (A), $50 \mu \mathrm{m}$. 

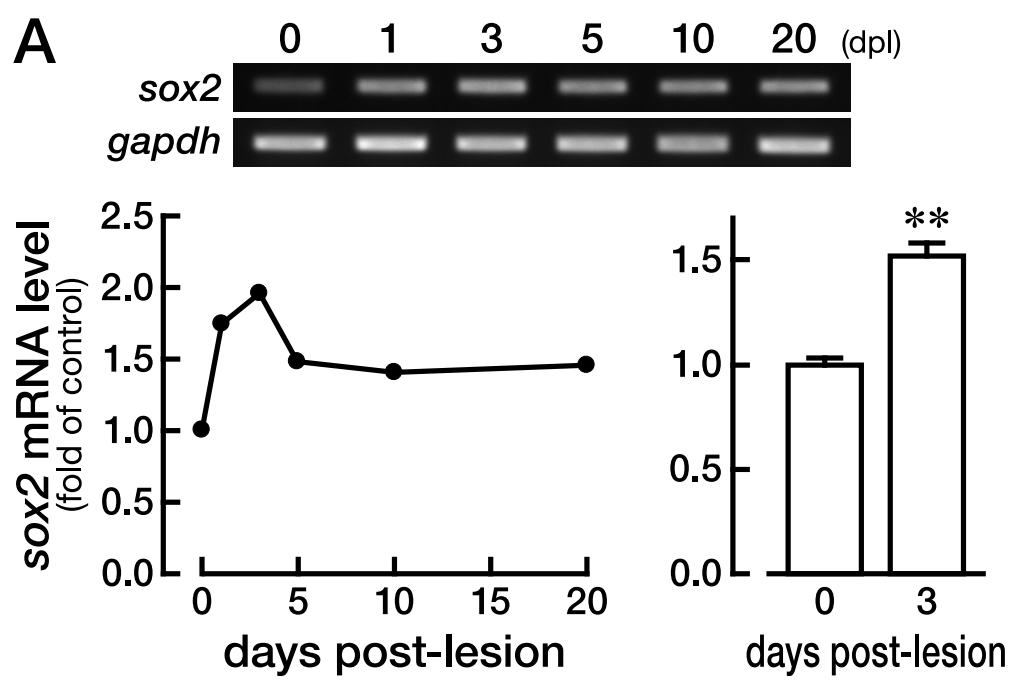

B

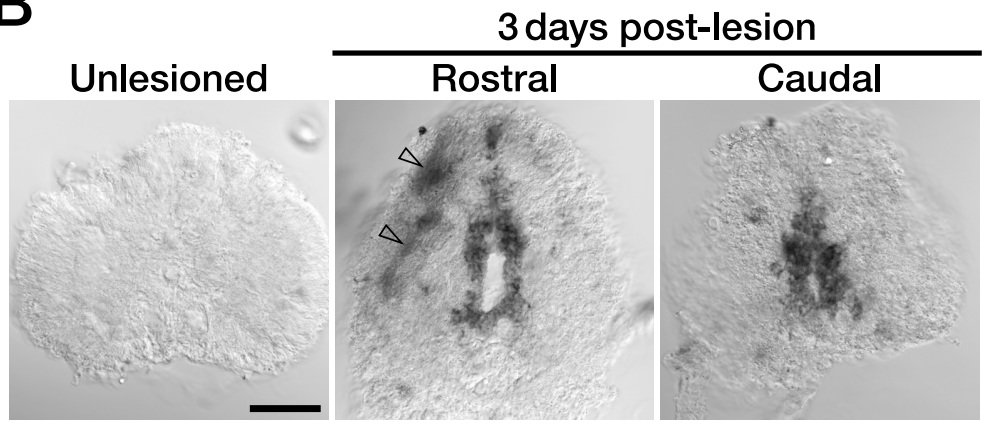

C 3 days post-lesion
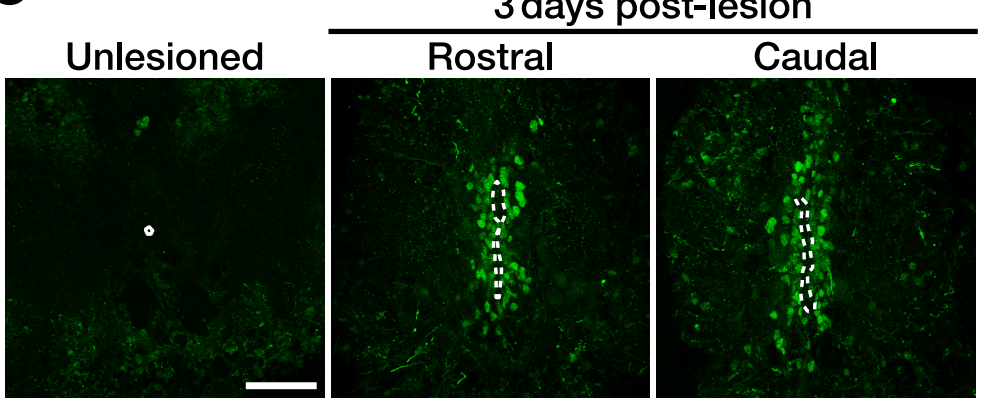

Fig. 1 Ogai et al. 


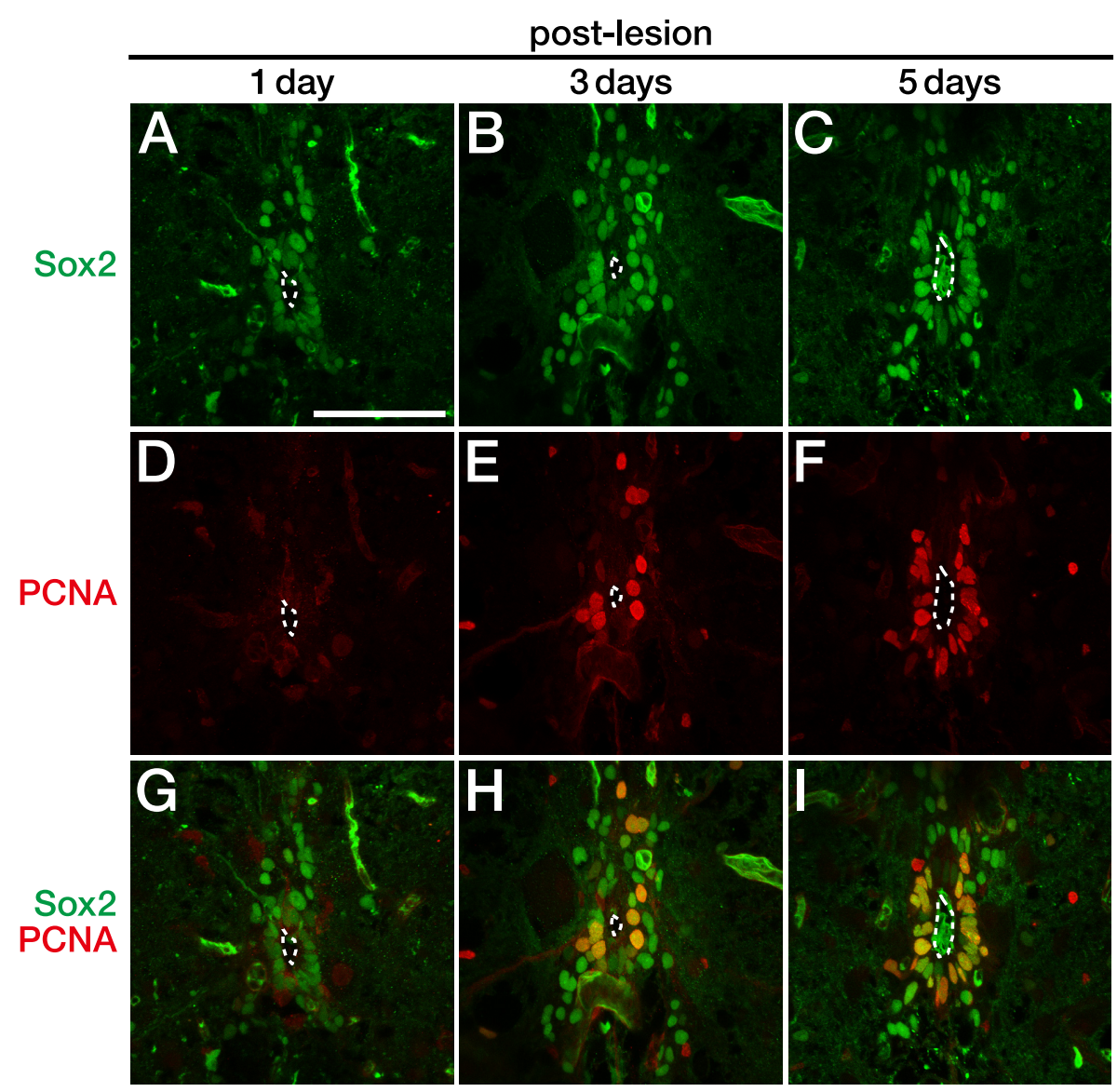

Fig. 2 Ogai et al. 


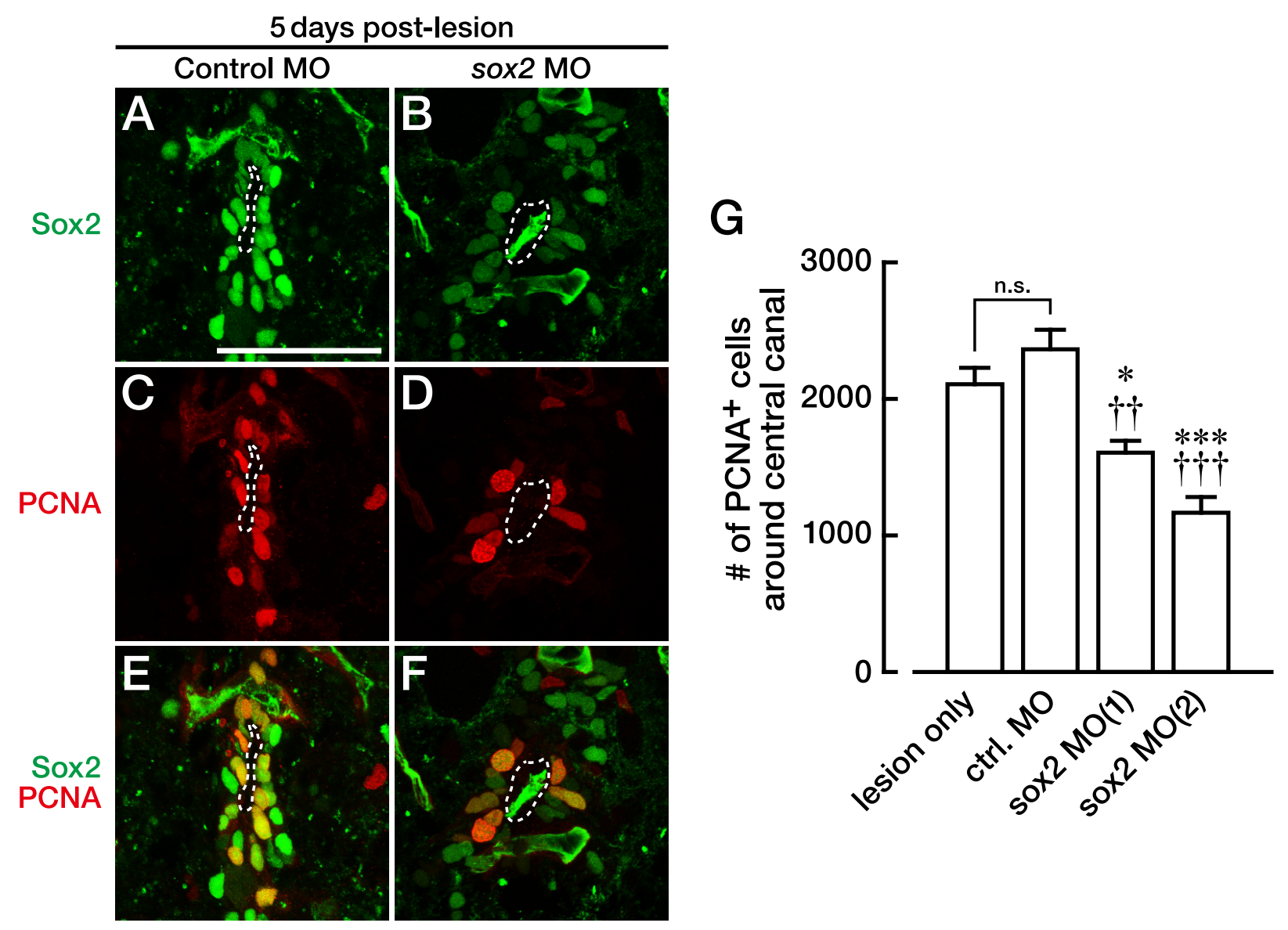

Fig. 3 Ogai et al. 